\title{
The Central Star of NGC 7027
}

N.A. Walton and S.R. Pottasch

Kapteyn Laboratorium

Groningen

The Netherlands

\author{
N.K. Reay \\ Queensgate Instruments Ltd. \\ Sunbury \\ Great Britain
}

T. Spoelstra

Radiosterrenwacht Dwingeloo

The Netherlands

\begin{abstract}
We have detected the central star of NGC 7027 by imaging the nebula through a narrow band 'continuum' filter onto the IPCS detector at the $2.5 \mathrm{~m}$ Isaac Newton Telscope. We obtain an apparent visual magnitude for the central star of $\mathrm{m}_{\mathrm{v}}=17.7 \pm 0.5$ mags.

Assuming that the central star radiates approximately as a blackbody, which is reasonable for the case of a hot star, then Zanstra temperatures for the central star can be calculated. We find $\mathrm{T}_{\mathrm{Z}}(\mathrm{H})=3.9 \times 10^{5} \mathrm{~K}$ and $\mathrm{T}_{\mathrm{Z}}(\mathrm{HeII})=2.6 \times 10^{5} \mathrm{~K}$. Using the correction due to Stasinska \& Tylenda (1986) we estimate the central star of NGC 7027 to have a temperature, $\mathrm{T}_{\text {eff }}=3.1 \times 10^{5} \mathrm{~K}$.

The luminosity and radii are found assuming a distance of $d=1.2 \mathrm{kpc}$., giving $\mathrm{L}=12,600 \mathrm{~L}_{\odot}$ and $R=0.039 R_{\odot}$. Placing the central star on the Log $L-\log T$ diagram and comparing with evolutionary tracks for central stars with various masses from Wood \& Faulkner (1986), indicates that the central star of NGC 7027 must have a mass, $M \geq 0.8 \mathrm{M}_{\odot}$.

Radio observations of NGC 7027 have been taken using the Westerbork Radio Synthesis Telesocpe at $21 \mathrm{~cm}$. Self calibration techniques have been employed to give a radio continuum map of high dynamic range. These observations are being compared with a deep optical $\mathrm{H} \beta$ map to study the nature of the faint halo seen around NGC 7027 (Atherton et al. 1979)
\end{abstract}

\section{References}

Atherton, P.D., Hicks, T.R., Reay, N.K., Robinson, G.J., Worswick, S.P., Phillips, J.P.:

1979, Astron. Astrophys. 232, 786

Stasinska, G., Tylenda, R.: 1986, Astron. Astrophys. 155, 137

Wood, P.R., Faulkner, D.J.: 1986, Astrophys. J. 307, 659

\section{Note Added In Proof}

Prime focus CCD observations of NGC 7027 have been obtained in Oct. 1987. These observations clearly show the central star. The results are presented in a forthcoming paper, Walton et al. 1988, Astron. Astrophys. Letts., accepted. 